\title{
Fuzzy Inference Systems in MR Image Processing-A Review
}

\author{
X.X. Yin, B. W.-H. Ng, D. Abbott, Fellow, IEEE \\ School of Electrical \& Electronic Engineering, \\ The University of Adelaide, \\ SA 5005, Australia \\ e-mail: xxyin@eleceng.adelaide.edu.au \\ * Yin is also with \\ Computer \& Network Engineering and School of \\ Electrical and Computer Engineering, Royal Melbourne \\ Institute of Technology, VIC 3001, Australia
}

\author{
W. Jia \\ Faculty of Engineering and Information Technology, \\ University of Technology, Sydney (UTS) \\ PO Box 123, Broadway, NSW, 2007, Australia \\ e-mail: wejia@it.uts.edu.au \\ X.X. Yin, K. Ramamohanarao \\ Department of Computer Science \& Software \\ Engineering, \\ The University of Melbourne, \\ VIC 3053, Australia \\ e-mail: rkotagiri@gmail.com
}

\begin{abstract}
Fuzzy inference systems are of great interest to provide a consistent mathematical framework for the representation of imprecision in relation to objects, relationships, knowledge and aims, and are viewed as powerful tools for reasoning and decision-making. In this paper, we survey several fuzzy approaches in magnetic resonances image processing, with an aim to develop and validate multidimensional segmentation and filtering methodology for future research. We also briefly review a number of advances of fuzzy set theory in the MR image processing application domain.
\end{abstract}

Keywords- fuzzy inference systems, MR image, deformable models, affinity, active contours, gradient vector

\section{INTRODUCTION}

Application of fuzzy set theory is making strong inroads in image processing application domain. This can be explained by its ability to model the inherent imprecision of images together with expert knowledge, and also by the availability of large and powerful tools for dealing with spatial information under imprecision [1], [2].

The analysis of brain magnetic resonance images (MRI) is a typical example of imaging application in which spatial relations of the image space (2D or $3 \mathrm{D})$, i.e. directional and distance relations are considered [3]. According to neuroanatomy [4], the human brain has a structured form and spatial relations are ubiquitous that are responsible for various congestive process. Regarding the characteristics of brain structures, relations between the structures themselves are constant among individual subjects and relatively independent of the acquisition parameters. Fuzzy sets constitute an appealing framework for representing spatial relations. Indeed, since spatial relations correspond to, for example, linguistic propositions, imprecision has to be taken into account, and fuzzy sets allow modeling of this imprecision [3]. In this framework, the strength of a given relation is represented by membership degree rather than in an all-or-nothing manner. Moreover, fuzzy sets can represent imprecision of objects, relationships, knowledge and aims, and provide a flexible framework for information fusion, reasoning and decision-making [5]. The fuzzy set framework has been used to represent different types of spatial relations including adjacencies [6], distances [7], directions [8], symmetries [9] and other complex relationships [10].

MRI at high magnetic fields $(>3.0 \mathrm{~T})$ is complicated by strong inhomogeneous radio-frequency fields, termed the "bias field" [11]. These lead to non-biological intensity nonuniformities across the image. These specific artifacts are still only partially corrected. With regards to the images to process and analyze, these artifacts translate into additive or multiplicative noise, and geometric or intensity distortions that drastically can affect the visual interpretation, and also complicate further image analysis such as registration, tissue segmentation, and the quantitative measures that follow [11].

The task of retrospectively correcting intensity inhomogeneity [12], [13], [14] in MRI scans has been extensively explored in the image processing literature over the past ten years. The problem and its solutions can be divided into two broad classes: methods aimed at robustly removing severe distortions with larger dynamic range, and methods aimed at accurately removing subtle intensity variations for improving identification of tissue types for quantisation.

This review paper consists of three basic sections. Section II reviews 3D brain segmentation in MRI using fuzzy spatial relations. Section III overviews the fuzzy restoration of MRI intensity distortion and intensity non-uniformities. Section IV presents suggested future work and Section $\mathrm{V}$ is the conclusion.

\section{FuZZY SPATIAL RELATIONSHIPS IN SEGMENTATION OF MRI}

\section{A. Fuzzy distances}

Fuzzy set methods have been used to model and manage uncertainty in various aspects of image processing, pattern recognition, and computer vision [15]. A classification of fuzzy distances is introduced in [7], which is needed for 
applications in image processing under imprecision. The distances (between two fuzzy sets) are measured among segments representing internal structures in brain MR images. A reference object, i.e. right ventricle (v2), is applied to compute the distances from all the structures of human brain. There are several main fuzzy approaches to defining spatial relationships which can be grouped into topological (set relationships, adjacency) and metrical (distances, directional relative positions) relations. Applications of such distances cover numerous fields, including image registration, assessment of relationships between image components, comparison of imprecise image objects and structural pattern recognition [5].

\section{B. Membership connectedness}

Fuzzy connectedness and fuzzy clustering are two wellknown techniques for fuzzy image segmentation. The former considers the relation of pixels in the spatial domain but does not inherently utilize their feature information. On the other hand, the latter does not consider the spatial relations among pixels [16]. A notion called membership connectedness has been proposed to combine these methods.

In this algorithm, two methods are involved in fuzzy treatment of geometric and topological concepts in image segmentation [17]. The first approach applies a fuzzy image segmentation technique to obtain a fuzzy subset wherein every pixel is assigned a fuzzy object membership grade and then defines the geometric and topological concepts on this fuzzy subset. The second approach develops these concepts directly on the given image, integrating with segmentation process.

An affinity function is defined [17], which is very critical in applications such as analysis of MR images. The performance of the proposed method is evaluated using a developed synthetic image dataset and both simulated and real brain MRI datasets. The evaluation demonstrates the strength of the proposed algorithm in segmentation of noisy images. It plays an especially important role in medical image applications.

\section{Deformable model and fuzzy spatial relations}

Deformable models offer a promising and vigorously researched computer-assisted medical image analysis technique [18]. Deformable models [19], also called active contours or snakes, are curves or surfaces evolving within an image from a starting point to a final state that should correspond to the target object, i.e. being segmented. Two types of information drive the evolution: a data term that attracts the model towards the edges of the image and a regularization term that forces the model to stay smooth and regular [18], [20]. They have proven to be effective in segmenting, matching, and tracking anatomic structures by exploiting constraints derived from the image data together with a priori knowledge about the location, size, and shape of these structures [3].
Based on spatial relations, the work carried out by [3] illustrates a general framework to integrate a type of constraints in deformable models. The aim is to use the framework to segment subcortical structures in brain MRI. In the proposed approach, spatial relations are represented as fuzzy subsets of the image space and incorporated in the deformable model as a new external "force". Let $\vec{v}$ be a gradient vector flow (GVF), $P$ be a point of the space, and $R$ be a fuzzy set representing a spatial relation (or a combination of relations), three methods can be used to construct an external force from a fuzzy set representing a spatial relation:

(1) An energy potential directly from the fuzzy set

$$
F_{R}^{1}(P)=-\left(1-\mu_{R}(P)\right) \nabla P_{R}^{1}(P) /\left\|\nabla P_{R}^{1}\right\|
$$

and

$P_{R}^{1}(P)=-\left(1-\mu_{R}(P)\right)+d_{\sup (R)}(P)$

where $d_{\sup (R)}$ is the distance from the support of $R$, a fuzzy set representing a spatial relation and $\mu_{k}$ is its membership function, which presents the degree of the spatial relation.

(2) A distance potential force

Distance potential forces [21], defining a potential as a function of a distance map to a binary edge detector, provide a broad attraction range

$$
F_{R}^{2}(P)=\nabla P_{R}=\nabla P_{R}^{2}(P)=\nabla g\left(d_{\mathrm{Ker}(R)}(P)\right)_{(3)}
$$

where $d_{\operatorname{ker}(R)}(p)$ is a distance map to the kernel of $R$ and $\nabla$ is the gradient operator.

\section{(3) A gradient diffusion technique}

The GVF, introduced in [22], computes a smooth vector field by diffusion technique. A constant term $c$ is used to maintain original gradient nearly unchanged in the region of high values. The GVF is the vector and $v$ satisfies the equation

$$
\left\{\begin{array}{l}
\frac{\partial v}{\partial t}=c \nabla^{2} v-\left\|\nabla \mu_{R}\right\|^{2}\left(v-\nabla \mu_{R}\right) \\
v(p, 0)=\nabla \mu_{R}(P) .
\end{array}\right.
$$

With the force of zero in the kernel, the normalization is used $F_{R}^{3}(P)=\left(1-\mu_{R}\right) \frac{\vec{v}}{\|\vec{v}\|}$.

The combination of several relations will thus be represented by a single force, corresponding to the fuzzy set obtained by the fusion. Maximum and minimum distances are calculated for parameter learning in order to define the fuzzy spatial relation. The results demonstrate that spatial relations applied in a deformable model can substantially improve the segmentation with low contrast and ill-defined boundaries. 


\section{Application to brain tumor detection}

Brain tumor detection and segmentation in magnetic resonance images (MRI) is important for medical diagnosis. The work carried by [23] investigates cancer segmentation by performing a symmetry analysis, assuming that tumors are generally not symmetrically placed in both approximately symmetrical brain hemispheres. Hyperintensity tumor segmentation based on fuzzy probabilistic cmeans (FPCM) [24] is adopted. FPCM solves the noise sensitivity problem of fuzzy c-means (FCM) and overcomes the problem of coincident clusters of probabilistic c-means (PCM). The mentioned method is promising for inclusion in longitudinal studies, for analyzing the evolution of the tumors and their impact on surrounding structures.

\section{FUZZY FILTERS IN THE RESTORATION OF MRI}

The multiplicity of sensors used in medical imaging leads to different types of noise. Non informative noise can damage the image interpretation process and the performance of automatic analysis [25]. Several fuzzy approaches have been explored to restore corrupted MRI, including heart and brain MRI data.

\section{A.iltering heart MRI data}

A study performed by [26] proposes a novel postprocessing strategy based on phase-contrast magnetic resonance imaging (PC MRI). The algorithm applies active contours for automatic vessel segmentation and the segmented velocity field is filtered using multidimensional fuzzy adaptive vector median filtering. The MRI data is acquired from children born with single-ventricle congenital heart defects. It illustrates the ability of this algorithm to visualize and quantify hemodynamics and to identify patients at risk of heart failure.

Random noise, incorporated into the segmentation, results from a blurred vessel edge. To detect and replace this noise, a hybrid multichannel filter framework is proposed

$$
y(\vec{k})=\mu(\vec{k}) i(\vec{k})+(1-\mu(\vec{k})) M(\vec{k})
$$

where $\vec{k}(k 1, k 2)$ is the pixel coordinate vector, $I$ is the input vector, $\mu \in[0,1]$ is a continuous fuzzy membership to determine to what extent $I$ is a flow pixel, and $M$ is the chosen filter value for replacing the noisy component of the vector. Vector median filtering is proposed to analyze a segmented PC MRI dataset, a vector field with each data point having three components. Due to the physical characteristics of flow and noise, the parameters used for establishing these rules are: (i) Distance of the given pixel from the vector median within the interrogation window (DVM); (ii) Vector direction homogeneity (VDH); (iii) Vector field standard deviation (SD); (IV) Magnitude image intensity (MI). A set of general rules therefore can be defined to characterize flow and noise:

Rule 1: DVM is high for noise and low for flow

Rule 2: VDH is low for noise and high for flow
Rule 3: SD is high for noise and low for flow

Rule 4: MI is low for noise and high for flow

A membership function $\mu$ is then evaluated to determine the probability of a pixel being noise by fuzzy fusion.

\section{B. Filtering brain MRI data}

In addition, a switch mode fuzzy adaptive median filter (SMFAMF) can be found in [27]. The fuzzy adaptive median filter is presented for noise reduction in MR images corrupted with heavy impulse (salt \& pepper) noise, without destroying edges and details in the image. The research group later investigated a fuzzy adaptive median filter with adaptive membership parameters (FAMFAMP) [28] for the same problem. It was found to out-perform the SMFAMF in suppressing salt \& pepper noise and other impulse-type noises.

\section{FUTURE WORK}

Partition filtering architectures [29] integrated with a fuzzy ranking vector technique have potential for coping with the non-stationary statistics of image structures, and restoring distorted MRI. This can also be applied to segment MRI via fuzzy spatial relations. This design scheme consists of three main function modules: fuzzy-ranked reference filtering, structure partitioning and weighted filtering. The respective aims of these modules are: to minimize the impact of noise contamination; to determine the noise level of the contaminated pixel by resultant partition cells and to restore the corrupted center pixel value via weighted averaging operation according to the partition result.

\section{CONCLUSION}

This paper briefly presents the main fuzzy approaches to segmenting and restoring MRI. Fuzzy relationships are important for describing spatial reasoning, membership connectedness, and noise detection, under imprecision. Deformable models with shape constraints and fuzzy filtering techniques have been investigated to achieve segmentation of different objects and restoration of subtle intensity variations. A future direction of research has been identified from this review. More details and illustrative examples can be found in the referenced papers.

\section{ACKNOWLEDGMENT}

This work was supported in part by the Australian Research Councils (ARC) Discovery Project funding scheme (Project No. DP0988064).

\section{REFERENCES}

[1] I. Bloch, "Image information processing using fuzzy sets (invited conference)," in Proc. World Automation Congress, Soft Computing with Industrial Applications, Montpellier, France, May 1996, pp. 7984.

[2] I. Bloch, "On fuzzy distances and their use in image processing under imprecision," Pattern Recognition, vol. 32, no. 11, pp. 1873-1895, 1999. 
[3] O. Colliot, O. Camara, and I. Bloch, "Integration of fuzzy spatial relations in deformable models - Application to brain MRI segmentation," Pattern Recognition, vol. 39, no. 8, pp. 1401-1414, 2006.

[4] S. Waxman, Correlative Neuroanatomy, 24th ed., New York: McGraw-Hill, 2000

[5] I. Bloch, "Fuzzy spatial relationships for image processing and interpretation: a review," Image and Vision Computing, vol. 23, no. 2, pp. 89-110, 2005

[6] I. Bloch, H. Maître, M. Anvari, Fuzzy adjacency between image objects, Int. J. Uncertainty Fuzziness Knowledge-Based Syst. vol. 5, no. 6, pp. 615-653, 1997

[7] I. Bloch, "On fuzzy distances and their use in image processing under imprecision," Patt. Recognition, Vol. 32, No.11, pp.1873-1895, 1999.

[8] I. Bloch, "Fuzzy relative position between objects in image processing: a morphological approach", IEEE Trans. Pattern Anal. Mach. Intell. Vol.21, No. 7, pp. 657-664, 1999.

[9] O. Colliot, A. Tuzikov, R. Cesar, I. Bloch, "Approximate reflectional symmetries of fuzzy objects with an application in model-based object recognition, " Fuzzy Sets Syst. Vol.147, No.1, pp. 141-163, 2004.

[10] I. Bloch, O. Colliot, R.M. Cesar, "On the ternary spatial relation " between" ," IEEE Trans. Syst. Man Cybern., Part B: Cybern. Vol. 36, No.2, pp. 312-327, 2006.

[11] S. Hadjidemetriou, C. Studholme, S. Mueller, M. Weiner, and N. Schuff, "Restoration of MRI data for intensity non-uniformities using local high order intensity statistics, " Medical Image Analysis, Vol. 13, No.1, pp. 36-48, 2009.

[12] C. Studholme, V. Cardenas, E. Song, F. Ezekiel, A. Maudsley, and M. Weiner, "Accurate template-based correction of brain MRI intensity distortion with application to dementia and aging," Medical Image Analysis, Vol. 23, No.1, pp. 99-110, 2004.

[13] J. G. Sled and G. B. Pike, "Understanding intensity non-uniformity in MRI," in Proc. MICCAI, 1998, pp. 614-622.

[14] J. G. Sled and B. Pike, "Standing-wave and RF penetration artifacts caused by elliptic geometry: An electrodynamic analysis of MRI," IEEE Trans. Med. Imag., vol. 17, pp. 653-662, Aug. 1998.

[15] P. Matsakis, J. M. Keller, L. Wendling, J. Marjamaa, and O. Sjahputera, "Linguistic Description of Relative Positions in Images," Medical Image Analysis, Vol. 31, No.4, pp. 573-588, 2001

[16] P. Matsakis, J. M. Keller, L. Wendling, J. Marjamaa, and O. Sjahputera, "Fuzzy Image Segmentation Using Membership Connectednes," EURASIP Journal on Advances in Signal Processing , Vol. 2008, Article ID 417293, 2008.

[17] J.K. Udupa, J.K.and P.K. Saha, "Fuzzy connectedness and image segmentation," Proc. of the IEEE, Vol. 91, No. 10, pp. 1649-1669, 2003

[18] T. McInerney and D.Terzopoulos, "Deformable Models in Medical Image Analysis: A Survey," Medical Image Analysis, vol. 1, No.2, pp. 91-108, 1996

[19] M. Kass, A. Witkin, D. Terzopoulos, "Snakes: active contour models," Int. J. Comput. Vision Vol.1 , No.4, pp. 321-331,1987

[20] C. Xu, D. Pham, J. Prince, "Medical image segmentation using deformable models, " in: J. Fitzpatrick, M. Sonka (Eds.), Handbook of Medical Imaging, vol. 2, SPIE Press, 2000, pp. 129-174

[21] L. Cohen, I. Cohen, "Finite element methods for active contour models and balloons for 2D and 3D images," IEEE Trans. Pattern Anal. Mach. Intell. Vol.15, No.11, pp. 1131-1147, 1993

[22] C. Xu and J. Prince, "Snakes, shapes and gradient vector flow," IEEETrans. Image Process. Vol.7, No.3, pp. 359-369, 1998.
[23] H. Khotanlou, O. Colliot, J. Atif, and I. Bloch, "3D brain tumor segmentation in MRI using fuzzy classification, symmetry analysis and spatially constrained deformable models," Fuzzy Sets and Systems, Vol. 160, No.10, pp.1457-1473, 2009

[24] N.R. Pal, K. Pal, J.C. Bezdek, "A mixed c-mean clustering model," in: IEEE Internat. Conf. on Fuzzy Systems, Vol. 1, 1997, pp. 11-21

[25] A. Ogier, P. Hellier, and C. Barillot, "Restoration of 3D medical images with total variation scheme on wavelet domains (TVW)," SPIE Medical Imaging 2006: Image Proc, San Diego, 2006, vol. 6144, pp. $465-473$

[26] K. S. Sundareswaran, D. H. Frakes, M.A. Fogel, D. D. Soerensen, J.N. Oshinski, A.P. Yoganathan "Optimum Fuzzy Filters for PhaseContrast Magnetic Resonance Imaging Segmentation," Journal of Magnetic Resonance Imaging, Vol. 29, No.1, pp.155-165, 2009

[27] A. Toprak, and İ. Güler, "Impulse noise reduction in medical images with the use of switch mode fuzzy adaptive median filter Source," Digital Signal Processing, vol. 17 No.4, pp. 711-723, July 2007.

[28] İ. Güüler, A. Toprak, A. Demirhan, and R. Karakış, "MR images restoration with the use of fuzzy filter having adaptive membership parameters," J. Med. Syst., vol. 32, No.3, pp. 229 - 234, July 2008.

[29] Z. Ma, H.R. Wu, and B. Qiu, "An structure adaptive hybrid vector filter for the restoration of digital color images," IEEE Transactions on Image Processing., vol. 14, No.12, pp. 1990 - 2001, 2005. 